\title{
Climate Variability and Predictability at Various Time Scales
}

\author{
Youmin Tang, ${ }^{1,2}$ Soon-Il An, ${ }^{3}$ and Wansuo Duan ${ }^{4}$ \\ ${ }^{1}$ Environmental Science and Engineering, University of Northern British Columbia, Prince George, BC, Canada V2N $4 Z 9$ \\ ${ }^{2}$ State Key Laboratory of Satellite Ocean Environment Dynamics, Second Institute of Oceanography, \\ State Oceanic Administration, Hangzhou, China \\ ${ }^{3}$ Department of Atmospheric Sciences, Yonsei University, 134 Shinchon-Dong, Seodaemun-Gu, Seoul, Republic of Korea \\ ${ }^{4}$ State Key Laboratory of Atmospheric Sciences and Geophysical Fluid Dynamics (LASG), \\ Institute of Atmospheric Physics Chinese Academy of Sciences, Beijing 100029, China
}

Correspondence should be addressed to Youmin Tang, ytang@unbc.ca

Received 2 December 2012; Accepted 2 December 2012

Copyright (C) 2012 Youmin Tang et al. This is an open access article distributed under the Creative Commons Attribution License, which permits unrestricted use, distribution, and reproduction in any medium, provided the original work is properly cited.

Climate change, variability, and predictability are a core component of climate dynamics. Recent advances in climate sciences have introduced new theories and technologies in detecting, diagnosing, analyzing, and predicting the climate variability on various time scales ranging from intraseasonal, seasonal, interannual to decadal-interdecadal time scales. This special issue is a small showcase of the efforts and progress made by international researchers in analyzing, diagnosing, and understanding climate change and climate variability at various time scales.

The six papers collected in this special issue can be roughly grouped into three topical categories. The first category detects the climate change signal and analyzes the possible forcing responsible for the climate change including natural forcing and anthropogenic forcing, focusing on the time scales from decades to centuries ( $\mathrm{S}$. Talento and $\mathrm{M}$. Barreiro; K. Zhuang and J. Giardino; X. Wang et al.). The second category focuses on seasonal and interannual climate variability (M. H. González et al.; S. Hameed and N. Riemer). Emphasis is placed on the analysis of local precipitation (Southern America and Sahel) and the mechanism responsible for these local climate variability at seasonal and interannual time scales. The third category addresses the potential predictability study for one of climate system components, the lake ecosystem, through sensitivity experiments of the growth of initial perturbation (X. Wang et al.). In particular, a novel nonlinear approach, called conditional nonlinear optimal perturbation (CNOP), was used here to carry out the sensitivity experiments. It has been argued that the CNOP is better than the conventional singular value (SV) method in characterizing the error growth of models of the initial perturbation.

This special issue is intended to promote the study of climate variability and predictability and to stimulate the continuing efforts to understand and predict climate variability on various time scales.

\section{Acknowledgments}

The publication of this issue would not be possible without the generous help and encouragement from the editorial board of Advances in Meteorology. We would like to thank the authors and reviewers for their contributions to this special issue.

Youmin Tang Soon-Il An Wansuo Duan 

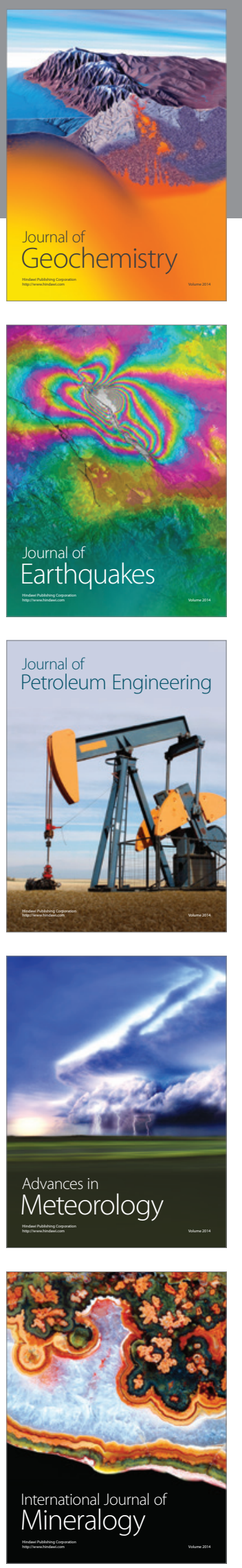
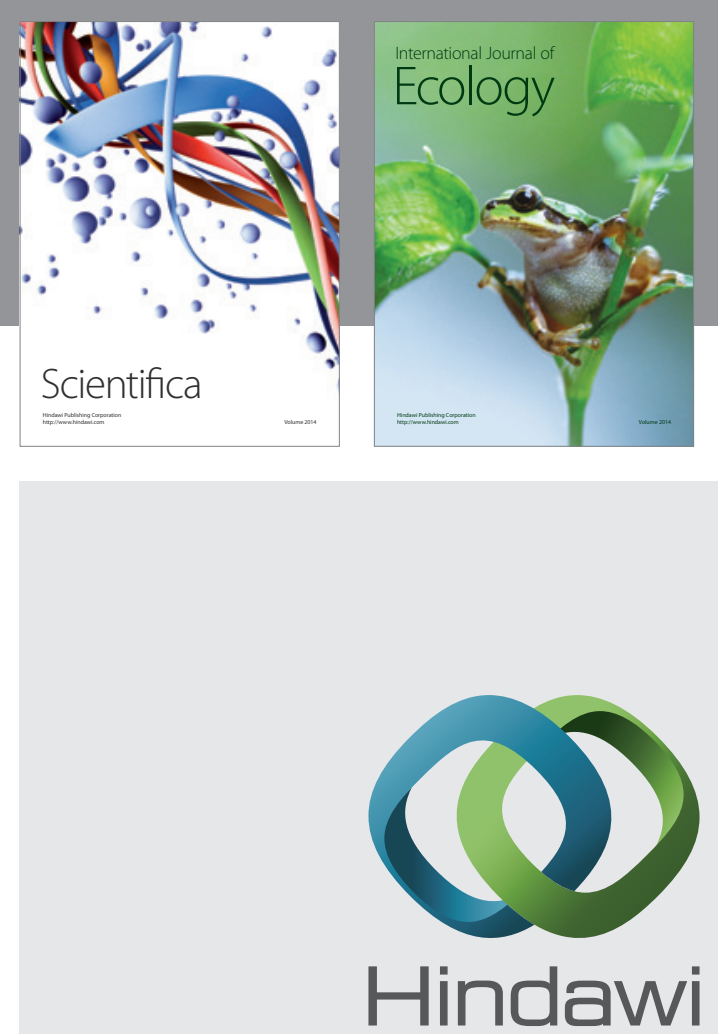

Submit your manuscripts at http://www.hindawi.com
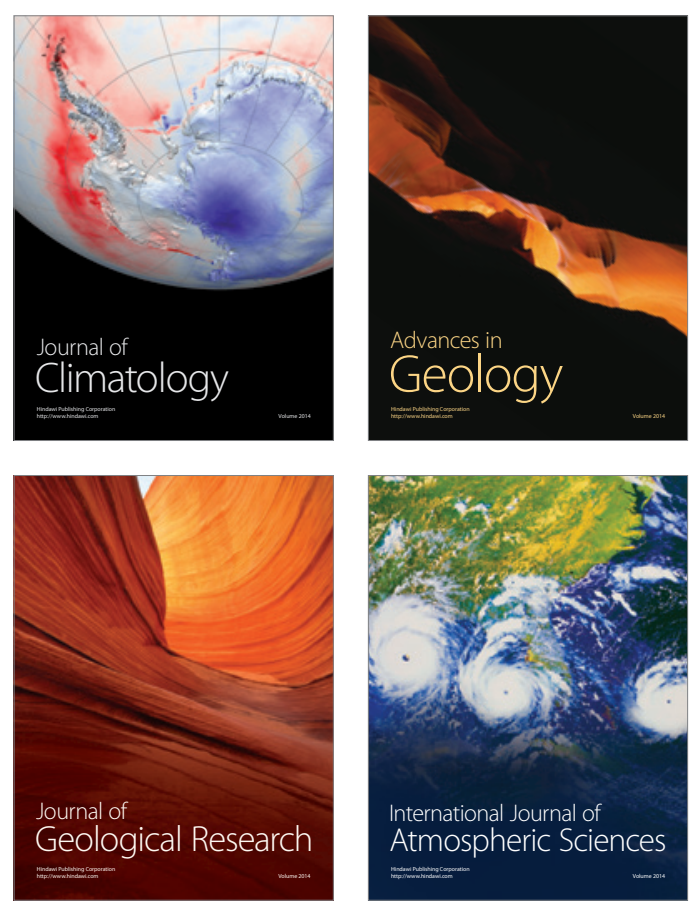
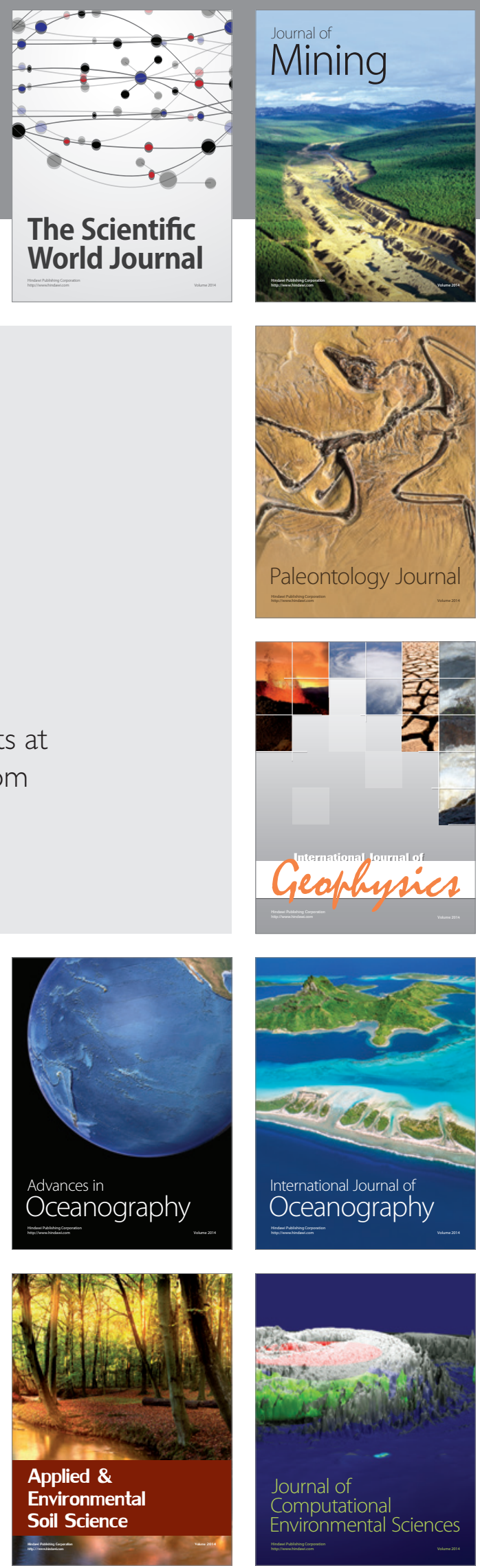